\title{
Test of the exponential decay law at short decay times using tau leptons
}

The OPAL Collaboration

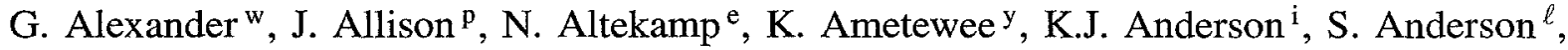
S. Arcelli ${ }^{b}$, S. Asai ${ }^{x}$, D. Axen ${ }^{\text {ac }}$, G. Azuelos ${ }^{\mathrm{r}, 1}$, A.H. Ball ${ }^{\mathrm{q}}$, E. Barberio ${ }^{\mathrm{z}}$, R.J. Barlow ${ }^{\mathrm{p}}$, R. Bartoldus ${ }^{\text {c }}$, J.R. Batley ${ }^{\mathrm{e}}$, G. Beaudoin ${ }^{\mathrm{r}}$, J. Bechtluft ${ }^{\mathrm{n}}$, G.A. Beck ${ }^{\mathrm{m}}$, C. Beeston ${ }^{\mathrm{p}}$, T. Behnke ${ }^{\mathrm{h}}$, A.N. Bell ${ }^{\mathrm{a}}$, K.W. Bell ${ }^{\mathrm{t}}$, G. Bella ${ }^{\mathrm{w}}$, S. Bentvelsen ${ }^{\mathrm{h}}$, P. Berlich ${ }^{\mathrm{j}}$, S. Bethke ${ }^{\mathrm{n}}$, O. Biebel ${ }^{\mathrm{n}}$, I.J. Bloodworth ${ }^{\mathrm{a}}$, J.E. Bloomer ${ }^{\mathrm{a}}$, P. Bock ${ }^{\mathrm{k}}$, H.M. Bosch ${ }^{\mathrm{k}}$, M. Boutemeur ${ }^{\mathrm{r}}$, B.T. Bouwens ${ }^{\ell}$, S. Braibant ${ }^{\ell}$, P. Bright-Thomas ${ }^{y}$, R.M. Brown ${ }^{t}$, H.J. Burckhart ${ }^{\mathrm{h}}$, C. Burgard $^{\text {aa }}$, R. Bürgin ${ }^{j}$, P. Capiluppi ${ }^{b}$, R.K. Carnegie ${ }^{f}$, A.A. Carter ${ }^{\mathrm{m}}$, J.R. Carter ${ }^{\mathrm{e}}$,

C.Y. Chang ${ }^{q}$, C. Charlesworth ${ }^{f}$, D.G. Charlton ${ }^{a, 2}$, D. Chrisman ${ }^{d}$, S.L. Chu ${ }^{d}$, P.E.L. Clarke ${ }^{\circ}$, S.G. Clowes $^{\text {p }}$, I. Cohen ${ }^{w}$, J.E. Conboy ${ }^{o}$, O.C. Cooke ${ }^{p}$, M. Cuffiani ${ }^{b}$, S. Dado ${ }^{v}$, C. Dallapiccola ${ }^{q}$, G.M. Dallavalle ${ }^{\mathrm{b}}$, C. Darling ${ }^{\text {ae }}$, S. De Jong ${ }^{\ell}$, L.A. del Pozo ${ }^{\mathrm{h}}$, M.S. Dixit ${ }^{g}$, E. do Couto e Silva ${ }^{\ell}$, E. Duchovni ${ }^{\mathrm{z}}$, G. Duckeck ${ }^{\mathrm{h}}$, I.P. Duerdoth ${ }^{\mathrm{p}}$, U.C. Dunwoody ${ }^{\text {h }}$, J.E.G. Edwards ${ }^{\text {, P.G. Estabrooks }}{ }^{\text {f }}$, H.G. Evans ${ }^{i}$, F. Fabbri ${ }^{b}$,

B. Fabbro ${ }^{\text {, }}$ P. Fath ${ }^{\mathrm{k}}$, F. Fiedler ${ }^{\ell}$, M. Fierro ${ }^{\mathrm{b}}$, M. Fincke-Keeler ${ }^{\mathrm{ab}}$, H.M. Fischer ${ }^{\mathrm{c}}$, R. Folman ${ }^{\mathrm{z}}$, D.G. Fong ${ }^{\mathrm{q}}$, M. Foucher ${ }^{\mathrm{q}}$, H. Fukui ${ }^{\mathrm{x}}$, A. Fürtjes ${ }^{\mathrm{h}}$, P. Gagnon ${ }^{\mathrm{g}}$, A. Gaidot ${ }^{\text {, }}$,

J.W. Gary ${ }^{\text {d, J. Gascon }}{ }^{\text {r }}$, S.M. Gascon-Shotkin ${ }^{\mathrm{q}}$, N.I. Geddes ${ }^{\mathrm{t}}$, C. Geich-Gimbel ${ }^{\mathrm{c}}$, S.W. Gensler ${ }^{i}$, F.X. Gentit ${ }^{u}$, T. Geralis ${ }^{t}$, G. Giacomelli ${ }^{b}$, P. Giacomelli ${ }^{d}$, R. Giacomelli ${ }^{b}$, V. Gibson ${ }^{\mathrm{e}}$, W.R. Gibson ${ }^{\mathrm{m}}$, D.M. Gingrich ${ }^{\text {ad,1, J. Goldberg }}{ }^{\mathrm{v}}$, M.J. Goodrick ${ }^{\mathrm{e}}$, W. Gorn ${ }^{\mathrm{d}}$, C. Grandi ${ }^{b}$, E. Gross ${ }^{\text {z }}$, C. Hajdu ${ }^{\text {af }}$, G.G. Hanson ${ }^{\ell}$, M. Hansroul ${ }^{\text {h }}$, M. Hapke ${ }^{\mathrm{m}}$, C.K. Hargrove g, P.A. Hart ${ }^{i}$, C. Hartmann ${ }^{c}$, M. Hauschild ${ }^{\text {h }}$, C.M. Hawkes ${ }^{\text {h }}$, R. Hawkings ${ }^{\text {h }}$, R.J. Hemingway ${ }^{f}$, G. Herten ${ }^{j}$, R.D. Heuer ${ }^{\text {, }}$, M.D. Hildreth ${ }^{h}$, J.C. Hill ${ }^{\mathrm{e}}$, S.J. Hillier ${ }^{\mathrm{h}}$, T. Hilse ${ }^{\text {j, P.R. Hobson }}{ }^{y}$, D. Hochman ${ }^{z}$, R.J. Homer ${ }^{a}$, A.K. Honma ${ }^{\text {ab,1 }}$, D. Horváth ${ }^{\text {af,3 }}$, R. Howard ${ }^{\text {ac }}$, R.E. Hughes-Jones ${ }^{\text {p }}$, D.E. Hutchcroft ${ }^{\mathrm{e}}$, P. Igo-Kemenes ${ }^{\mathrm{k}}$, D.C. Imrie ${ }^{\mathrm{y}}$, A. Jawahery ${ }^{q}$, P.W. Jeffreys ${ }^{t}$, H. Jeremie ${ }^{r}$, M. Jimack ${ }^{a}$, A. Joly ${ }^{r}$, M. Jones ${ }^{f}$, R.W.L. Jones ${ }^{\mathrm{h}}$, U. Jost ${ }^{\mathrm{k}}$, P. Jovanovic ${ }^{\mathrm{a}}$, D. Karlen ${ }^{\mathrm{f}}$, T. Kawamoto ${ }^{\mathrm{x}}$, R.K. Keeler ${ }^{\mathrm{ab}}$, R.G. Kellogg ${ }^{\mathrm{q}}$, B.W. Kennedy ${ }^{\mathrm{t}}$, B.J. King ${ }^{\mathrm{h}}$, J. King ${ }^{\mathrm{m}}$, J. Kirk ${ }^{\mathrm{ac}}$, S. Kluth ${ }^{\mathrm{e}}$, T. Kobayashi ${ }^{\mathrm{x}}$, M. Kobel ${ }^{j}$, D.S. Koetke ${ }^{\mathrm{f}}$, T.P. Kokott ${ }^{\mathrm{c}}$, S. Komamiya ${ }^{\mathrm{x}}$, R. Kowalewski ${ }^{\mathrm{h}}$, T. Kress ${ }^{\mathrm{k}}$,

P. Krieger ${ }^{f}$, J. von Krogh ${ }^{k}$, P. Kyberd ${ }^{m}$, G.D. Lafferty ${ }^{p}$, H. Lafoux ${ }^{u}$, R. Lahmann ${ }^{q}$, W.P. Lai ${ }^{\text {s }}$, D. Lanske ${ }^{\text {n }}$, J. Lauber ${ }^{\circ}$, J.G. Layter ${ }^{d}$, A.M. Lee ${ }^{\text {ae }}$, E. Lefebvre ${ }^{r}$, D. Lellouch ${ }^{z}$, 
J. Letts ${ }^{b}$, L. Levinson ${ }^{z}$, C. Lewis ${ }^{o}$, S.L. Lloyd ${ }^{m}$, F.K. Loebinger ${ }^{p}$, G.D. Long ${ }^{q}$, B. Lorazo ${ }^{\mathrm{r}}$, M.J. Losty ${ }^{\mathrm{g}}$, J. Ludwig ${ }^{\mathrm{j}}$, A. Luig ${ }^{\mathrm{j}}$, A. Malik ${ }^{\mathrm{u}}$, M. Mannelli ${ }^{\mathrm{h}}$, S. Marcellini ${ }^{\mathrm{b}}$, C. Markus ${ }^{\text {c }}$, A.J. Martin ${ }^{\mathrm{m}}$, J.P. Martin ${ }^{\mathrm{r}}$, G. Martinez ${ }^{\mathrm{q}}$, T. Mashimo ${ }^{\mathrm{x}}$, W. Matthews ${ }^{\mathrm{y}}$, P. Mättig ${ }^{c}$, W.J. McDonald ${ }^{\text {ad }}$, J. McKenna ${ }^{\text {ac }}$, E.A. Mckigney ${ }^{\circ}$, T.J. McMahon ${ }^{a}$, A.I. $\mathrm{McNab}^{\mathrm{m}}$, F. Meijers ${ }^{\mathrm{h}}$, S. Menke ${ }^{\text {c }}$, F.S. Merritt ${ }^{\mathrm{i}}$, H. Mes ${ }^{\mathrm{g}}$, J. Meyer ${ }^{\text {aa }}$, A. Michelini ${ }^{\mathrm{h}}$, G. Mikenberg ${ }^{\mathrm{z}}$, D.J. Miller ${ }^{\circ}, \mathrm{R}_{\text {Mir }}{ }^{\mathrm{z}}$, W. Mohr ${ }^{\mathrm{j}}$, A. Montanari ${ }^{\mathrm{b}}$, T. Mori ${ }^{\mathrm{x}}$, M. Morii ${ }^{\mathrm{x}}$, U. Müller ${ }^{c}$, B. Nellen ${ }^{c}$, B. Nijjhar ${ }^{p}$, R. Nisius ${ }^{\text {h }}$, S.W. O'Neale ${ }^{\text {a }}$, F.G. Oakham ${ }^{\mathrm{g}}$, F. Odorici ${ }^{b}$, H.O. Ogren ${ }^{\ell}$, N.J. Oldershaw ${ }^{\text {p }}$, T. Omori ${ }^{x}$, C.J. Oram ${ }^{a b, 1}$, M.J. Oreglia ${ }^{i}$, S. Orito ${ }^{\mathrm{x}}$, M. Palazzo ${ }^{\mathrm{b}}$, J. Pálinkás ${ }^{\mathrm{ag}}$, F.M. Palmonari ${ }^{\mathrm{b}}$, J.P. Pansart ${ }^{\mathrm{u}}$, G. Pásztor ${ }^{\mathrm{ag}}$, J.R. Pater $^{\text {p}}$, G.N. Patrick ${ }^{\text {t }}$, M.J. Pearce ${ }^{\text {a }}$, P.D. Phillips ${ }^{\text {p }}$, J.E. Pilcher ${ }^{i}$, J. Pinfold ${ }^{\text {ad }}$, D.E. Plane ${ }^{\text {h }}$, P. Poffenberger ${ }^{\text {ab }}$, B. Poli ${ }^{b}$, A. Posthaus ${ }^{c}$, T.W. Pritchard ${ }^{m}$, H. Przysiezniak ${ }^{\text {ad }}$,

D.L. Rees ${ }^{a}$, D. Rigby ${ }^{a}$, M.G. Rison ${ }^{\mathrm{c}}$, S.A. Robins ${ }^{\mathrm{m}}$, N. Rodning ${ }^{\text {ad }}$, J.M. Roney ${ }^{\mathrm{ab}}$, E. Ros ${ }^{\text {h }}$, A.M. Rossi ${ }^{b}$, M. Rosvick ${ }^{\text {ab }}$, P. Routenburg ad, Y. Rozen ${ }^{\text {h }}$, K. Runge ${ }^{\mathrm{j}}$, O. Runolfsson ${ }^{\text {h}}$, D.R. Rust ${ }^{\ell}$, R. Rylko ${ }^{y}$, E.K.G. Sarkisyan ${ }^{w}$, M. Sasaki ${ }^{x}$, C. Sbarra ${ }^{b}$, A.D. Schaile ${ }^{\text {h }}$, O. Schaile ${ }^{j}$, F. Scharf ${ }^{c}$, P. Scharff-Hansen ${ }^{\text {h}}$, P. Schenk ${ }^{d}$, B. Schmitt ${ }^{\text {c }}$, M. Schröder ${ }^{h}$, H.C. Schultz-Coulon ${ }^{j}$, M. Schulz ${ }^{h}$, P. Schütz ${ }^{c}$, J. Schwiening ${ }^{c}$, W.G. Scott ${ }^{t}$, T.G. Shears ${ }^{p}$, B.C. Shen ${ }^{d}$, C.H. Shepherd-Themistocleous ${ }^{\text {aa }}$, P. Sherwood ${ }^{\circ}$, G.P. Siroli ${ }^{b}$, A. Sittler ${ }^{\text {aa }}$, A. Skillman ${ }^{\circ}$, A. Skuja ${ }^{\text {q }}$, A.M. Smith ${ }^{\text {h }}$, T.J. Smith ${ }^{\text {ab }}$, G.A. Snow ${ }^{\text {q }}$, R. Sobie ${ }^{\text {ab }}$, S. Söldner-Rembold ${ }^{\mathfrak{j}}$, R.W. Springer ${ }^{\text {ad }}$, M. Sproston ${ }^{\mathrm{t}}$, A. Stahl ${ }^{\mathrm{c}}$, M. Starks ${ }^{\ell}$, C. Stegmann ${ }^{\mathrm{j}}$, K. Stephens ${ }^{\text {P }}$, J. Steuerer ${ }^{\mathrm{ab}}$, B. Stockhausen ${ }^{\text {c }}$, D. Strom ${ }^{\text {s }}$, F. Strumia ${ }^{\text {h }}$, P. Szymanski ${ }^{\mathrm{t}}$,

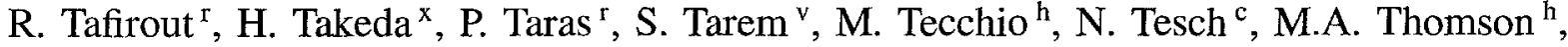
E. von Törne ${ }^{c}$, S. Towers ${ }^{f}$, M. Tscheulin ${ }^{\mathrm{j}}$, T. Tsukamoto ${ }^{\mathrm{x}}$, E. Tsur ${ }^{\mathrm{w}}$, A.S. Turcot ${ }^{\mathrm{i}}$, M.F. Turner-Watson ${ }^{h}$, P. Utzat ${ }^{k}$, R. Van Kooten ${ }^{\ell}$, G. Vasseur ${ }^{\mathrm{u}}$, P. Vikas ${ }^{\mathrm{r}}$, M. Vincter ${ }^{\text {ab }}$, E.H. Vokurka ${ }^{\mathrm{p}}$, F. Wäckerle ${ }^{\mathrm{j}}$, A. Wagner ${ }^{\text {aa }}$, D.L. Wagner ${ }^{\mathrm{i}}$, C.P. Ward ${ }^{\mathrm{e}}$, D.R. Ward ${ }^{\mathrm{e}}$, J.J. Ward ${ }^{\mathrm{o}}$, P.M. Watkins ${ }^{\mathrm{a}}$, A.T. Watson ${ }^{\mathrm{a}}$, N.K. Watson ${ }^{\mathrm{g}}$, P. Weber ${ }^{\mathrm{f}}$, P.S. Wells ${ }^{\mathrm{h}}$, N. Wermes ${ }^{\text {c }}$, B. Wilkens ${ }^{j}$, G.W. Wilson ${ }^{\text {aa }}$, J.A. Wilson ${ }^{a}$, T. Wlodek ${ }^{z}$, G. Wolf ${ }^{z}$, S. Wotton ${ }^{\mathrm{k}}$, T.R. Wyatt ${ }^{\mathrm{p}}$, S. Xella ${ }^{\mathrm{b}}$, S. Yamashita ${ }^{\mathrm{x}}$, G. Yekutieli ${ }^{\mathrm{z}}$, V. Zacek ${ }^{\mathrm{r}}$

a School of Physics and Space Research, University of Birmingham, Birmingham B15 2TT, UK

${ }^{\mathrm{b}}$ Dipartimento di Fisica dell' Universita di Bologna and INFN, I-40126 Bologna, Italy c Physikalisches Institut, Universität Bonn, D-53115 Bonn, Germany

d Department of Physics, University of California, Riverside, CA 92521, USA - Cavendish Laboratory, Cambridge CB3 OHE, UK

'Ottawa-Carleton Institute for Physics, Department of Physics, Carleton University, Ottawa, Ont. K1S 5B6, Canada

g Centre for Research in Particle Physics, Carleton University, Ottawa, Ont. KIS 5B6, Canada

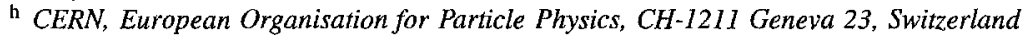

i Enrico Fermi Institute and Department of Physics, University of Chicago, Chicago, IL 60637, USA

j Fakultät für Physik, Albert Ludwigs Universität, D-79104 Freiburg, Germany

k Physikalisches Institut, Universität Heidelberg, D-69120 Heidelberg, Gernany

'Indiana University, Department of Physics, Swain Hall West 117, Bloomington, IN 47405, USA

m Queen Mary and Westfield College, University of London, London E1 4NS, UK

${ }^{n}$ Technische Hochschule Aachen, III Physikalisches Institut, Sommerfeldstrasse 26-28, D-52056 Aachen, Germany - University College London, London WC1E 6BT, UK

p Department of Physics, Schuster Laboratory, The University, Manchester MI3 9PL, UK q Department of Physics, University of Maryland, College Park, MD 20742, USA

${ }^{r}$ Laboratoire de Physique Nucléaire, Université de Montréal, Montréal, Que. H3C 3J7, Canada 


\author{
s University of Oregon, Department of Physics, Eugene OR 97403, USA \\ ${ }^{1}$ Rutherford Appleton Laboratory, Chilton. Didcot, Oxfordshire OXI1 OQX, UK \\ " CEA, DAPNIA/SPP, CE-Saclay, F-91191 Gif-sur-Yvette, France \\ ${ }^{\vee}$ Department of Physics, Technion-Israel Institute of Technology, Haifa 32000, Israel \\ w Department of Physics and Astronomy, Tel Aviv University, Tel Aviv 69978, Israel \\ x International Centre for Elementary Particle Physics and Department of Physics, University of Tokyo, Tokyo 113, Japan \\ and Kobe University, Kobe 657, Japan \\ y Brunel University, Uxbridge, Middlesex UB8 $3 P H, U K$ \\ ${ }^{2}$ Particle Physics Department, Weizmann Institute of Science, Rehovot 76100, Israel \\ aa Universität Hamburg/DESY, II Institut für Experimental Physik, Notkestrasse 85, D-22607 Hamburg, Germany \\ ab University of Victoria, Department of Physics. P.O. Box 3055, Victoria BC V8W 3P6. Canada \\ ${ }^{a c}$ University of British Columbia, Department of Physics, Vancouver BC V6T 1ZI, Canada \\ ad University of Alberta, Department of Physics, Edmonton AB T6G 2J1. Canada \\ ae Duke University, Department of Physics, Durham, NC 27708-0305, USA \\ af Research Institute for Particle and Nuclear Physics, H-1525 Budapest, P.O. Box 49, Hungary \\ ag Institute of Nuclear Research, H-4001 Debrecen, P.O. Box 51, Hungary
}

Received 8 November 1995

Editor: K. Winter

\begin{abstract}
Quantum mechanics predicts an exponential distribution for the decay time of massive particles. However, deviations are expected for decay times shorter than about $10^{-13} \mathrm{~s}$ in models conjecturing the existence of hidden variables. Following a recent proposal, the decay length distribution of $5843 \tau$ leptons decaying into 3 charged particles was analyzed in search of such a deviation. The deviation from an exponential distribution with respect to the number of decays present within the exponential form, expressed as the relative weight of an excess at zero decay length, was measured to be $1.1 \% \pm 1.4 \% \pm$ $3.5 \%$. This result is consistent with zero deviation and leads to an upper limit of $8.5 \%$ and a lower limit of $-6.3 \%$ at the $95 \%$ confidence level.
\end{abstract}

Theories exist which suggest that the shape of the decay time distribution of unstable particles is not exponential. The Zeno effect [1], for example, maintains that an unstable particle, when monitored at sufficiently small intervals of time, will live longer than a particle monitored infrequently. However, these effects are usually calculated to be apparent only in the very short or very long decay time regions (e.g. below $10^{-14} \tau_{0}$ or above $190 \tau_{0}$ [1], where $\tau_{0}$ is the lifetime). No deviations from the exponential decay law have been observed experimentally [2] and the decay distribution has always been assumed to be exponential.

Theories of hidden variables have been proposed as an alternative to quantum mechanics. For example, the theory of non-local hidden variables of the Bohm-

\footnotetext{
${ }^{1}$ Also at TRIUMF, Vancouver, Canada V6T $2 \mathrm{~A} 3$.

${ }^{2}$ Royal Society University Research Fellow.

${ }^{3}$ Institute of Nuclear Research, Debrecen, Hungary.
}

Bub type [3] suggests that the decay time distribution of massive particles may not be exponential [4] It states that quantum mechanics is a description of a thermodynamical equilibrium of the system. So, if a state occupying only a partial volume of the phase space is prepared, only measurements which are performed very quickly after its production will exhibit the pre-equilibrium effects. Otherwise, after a characteristic relaxation time (hypothesized by Bohm to be $\tau \approx 10^{-13} \mathrm{~s}[3]$ ) the equilibrium forces will fill phase space and yield normal quantum mechanical expectation values for any subsequent measurements. To observe the pre-equilibrium state a double measurement scheme is needed, with a small time interval between the measurements. Bohm suggested an experimental set-up capable of checking whether massive particles are influenced by the Bohm-Bub type of hidden variables, but this set-up has never been realized. Other experiments, such as those mentioned above [2], did 
not include the specific features needed to check for the existence of hidden variables of the Bohm-Bub type: two consecutive collapses with the correct time scale between them and more than one non-zero collapse channel in each decay.

In a recent proposal [4], motivated by the idea of hidden variables of the Bohm-Bub type, an alternative to the double measurement scheme was suggested using two sequential decays $-Z^{0}$ into $\tau^{+} \tau^{-}$and $\tau$ into three prongs. Only $\tau$ decays occurring very quickly after the $\tau$ has been formed are expected to have sensitivity to deviations from quantum mechanics. That is, deviations from the standard exponential distribution may occur at short decay times, corresponding to short decay lengths, though there is no precise prediction for the magnitude of the effect. An observed deviation from an exponential in one decay channel would open the possibility of time dependent branching ratios [4].

Following this proposal, we have analyzed three prong $\tau$ decays in search of an excess of decays with small decay lengths. The $\tau$ lepton was chosen because it is an elementary particle, its lifetime is of order $10^{-13} \mathrm{~s}$, its decays can be selected with almost no background at centre-of-mass energies near the $Z^{0}$ resonance and because its decay length to three prongs can be measurcd. This analysis is based on data collected in 1992 and 1993 with the OPAL detector at LEP, corresponding to an integrated luminosity of 58 $\mathrm{pb}^{-1}$, collected at centre-of-mass energies near the $Z^{0}$ peak. A complete description of the OPAL detector can be found elsewhere [5]. Of particular importance for this analysis is the silicon micro-vertex detector, which provides precise determination of track parameters in the $r-\phi$ plane [6].

The selection of $\tau$ pair events and the three-prong lifetime analysis are described in full in Ref. [7]. The major points of the lifetime analysis are that candidate three-prong decays were selected from the $\tau$ pair sample by requiring that a thrust hemisphere contain exactly three tracks of net charge \pm 1 . These three tracks are fitted to a vertex in the plane transverse to the beam axis and the three dimensional decay length is determined using the beam spot and the event thrust axis. The decay length is corrected to account for the variation in the average energy of the $\tau$ lepton at each centre of mass energy.

Since the analysis is very dependent on both a good resolution of the decay length measurement and a pre- cise knowledge of this resolution, some of the cuts are different from those of the lifetime analysis. Firstly, the mass of the three-prong candidate is required to be less than $1.8 \mathrm{GeV}$. Secondly, in order to only use events with precise silicon micro-vertex detector information, $\left|\cos \theta_{\text {thrust }}\right|$ is required to be less than 0.85 . At least one silicon detector hit per track is required, and each track is required to have a fit probability $>$ $0.1 \%$. Thirdly, the $\chi^{2}$ probability of the vertex fit must be greater than $5 \%$. Fourthly, the error on the decay length, typically $1 \mathrm{~mm}$, must be less than $3 \mathrm{~mm}$. Only three-prong candidates having decay length within the interval $[-15,+25 \mathrm{~mm}]$ are considered. This selection yields 5843 three-prong $\tau$ decays, which is approximately $40 \%$ of the total number of three-prong decays. The fraction of low multiplicity multi-hadron events in the $\tau$ sample is estimated to be $0.25 \pm 0.25 \%$. No other contamination is found.

To test for deviations from cxponcntial bchaviour, the decay length distribution is fitted with a likelihood function equal to the true decay length distribution smeared with a Gaussian resolution function. Specifically, there are three free fit parameters: $L$, the average decay length of the exponential component (in $\mathrm{mm}$ ), $S$, the decay length error scaling factor (for each event $i, \sigma_{i}$, the calculated error on the decay length, is replaced by $S \cdot \sigma_{i}$ ), and $D$, the deviation from the expected exponential, expressed as a fraction of the total number of such decays present within the exponential form. In a conventional lifetime analysis $D$ is zero. As all proposed deviation schemes point to the short decay time region, the decay length deviation, in the case of enhancement, is taken in the form of a delta function distribution at zero decay length. As a further check, a constant enhancement distribution between 0.0 and $0.5 \mathrm{~mm}$ was also examined, giving rise to similar results (the value of $0.5 \mathrm{~mm}$ corresponds to the $10^{-13} \mathrm{~s}$ time scale hypothesized by Bohim). Negative deviations $(D<0)$ are treated differently. To parametrize these deviations we take a form where the decay probability for events with decay length $l<l_{c}$ is zero, where $l_{c}$ is such that integrating from zero to $l_{c}$ over the expected exponential form yields $D$. We also use a compensating probability normalization procedure for the loss, due to decay length window cuts, of long lived $\tau$ candidates; for a given set of parameters $L, S$ and $D$, where $r$ is the expected fraction of events outside the window, we replace the full-window like- 
Table 1

Results of the two and three parameter fits to simulated events for different values of the deviation parameter $D$

\begin{tabular}{cllll}
\hline & Simulated $D$ & $-4 \%$ & 0 & $+4 \%$ \\
\hline \multirow{2}{*}{ 2-parameter fit } & $L(\mathrm{~mm})$ & $2.37 \pm 0.02$ & $2.30 \pm 0.02$ & $2.21 \pm 0.02$ \\
& $S$ & $0.937 \pm 0.011$ & $0.977 \pm 0.011$ & $1.000 \pm 0.011$ \\
& $L(\mathrm{~mm})$ & $2.31 \pm 0.02$ & $2.30 \pm 0.02$ & $2.27 \pm 0.02$ \\
3-parameter fit & $S$ & $0.961 \pm 0.012$ & $0.973 \pm 0.011$ & $0.964 \pm 0.012$ \\
& $D(\%)$ & $-2.6 \pm 0.6$ & $+0.4 \pm 0.5$ & $+3.2 \pm 0.7$ \\
\hline
\end{tabular}

lihood $p$ by $p /(1-r)$.

We have tested our ability to detect a deviation at zero decay length by applying the three-parameter fit to a sample of about 20000 events generated by the full detector Monte Carlo simulation described in [7]. The input lifetime was $303 \mathrm{fs}$ which corresponds to a mean decay length of $2.30 \mathrm{~mm}$. The fit returned the values shown in Table 1 .

The results of a 2-parameter fit demonstrate the sensitivity of the lifetime measurements to non-zero values of $D$. The imperfection in the reproduction of $D$ by the three-parameter fit is due to a strong correlation between $D$ and $S$. We have checked the detection power of the fit procedure on real data by adding a sample of zero decay-length fake $\tau$ 's to the selected $\tau$ sample. These are three-prong vertices which are selected from multi-hadron events and satisfy the $\tau$ decay selection cuts. The fit to the $\tau$ sample containing these fake $\tau$ vertices returns a $D$ value of $+11.9 \%$ $\pm 1.6 \%$ which is consistent with the additional zero decay length component of $9.2 \%$. Therefore, we conclude that within the limitations set by systematic and statistical uncertainties, we are able to detect a zero lifetime component.

Several sources of systematic errors have been considered:

- Dependence on accelerator operation mode and

\section{Table 2}

Dependence on angle and accelerator operation mode of the $D$ parameter

\begin{tabular}{lrrr}
\hline 1-parameter fit $(D$ in $\%)$ & & \\
\hline Running period & 92, part 1 & 92, part 2 & 93 \\
\hline$\left|\cos \phi_{\tau}\right|>0.707$ & $6.7 \pm 2.5$ & $7.2 \pm 4.5$ & $0.2 \pm 1.8$ \\
$\left|\cos \phi_{\tau}\right|<0.707$ & $-0.6 \pm 1.9$ & $0.7 \pm 3.5$ & $-3.8 \pm 1.7$ \\
\hline
\end{tabular}

event angle: We have examined our results as a function of time periods corresponding to changes in the accelerator operation mode. Because the beam spot in LEP possesses a high aspect ratio, we have also checked our method as a function of the azimuthal orientation of the event thrust axis $\phi_{\tau}$, by differentiating between events with $\left|\cos \phi_{\tau}\right|>0.707$ and $\left|\cos \phi_{\tau}\right|<0.707 . L$ and $S$ were fixed to their fitted values for the whole sample. The results are shown in Table 2. Computing the variance of the six values and subtracting the contribution of statistical fluctuations, we assign a $\pm 3.2 \%$ uncertainty to the value of $D$. This is the dominant systematic error.

- Misevaluation of decay length errors: Contributions to the decay length errors from tracking errors were investigated by changing the tracking errors in a reasonable range and noting the effect on the scale factor. These studies indicate a $5 \%$ uncertainty on $S$, corresponding to a $1.2 \%$ uncertainty on $D$. This error is consistent with the variations seen in $S$ in the subsamples of Table 2 . The sample of zero decay length three-prong vertices described above confirms that the errors on their decay lengths are well described.

- Beam-spot position uncertainties: Monte Carlo simulations show a depletion of the zero decay length region of the decay length distribution when the beam-spot position is mismeasured. One can evaluate the difference between the assigned and the actual beam spot positions by examining the time variations of the multi-hadronic events main vertex position inside a run. The accuracy of the assignment is conservatively estimated to be $50 \pm 50 \mu \mathrm{m}$ for both horizontal and vertical directions. This corresponds to a depletion in the zero decay length region of $0.5 \pm 0.5 \%$, and is used as a correction to 
Table 3

Summary of systematic uncertainties

\begin{tabular}{llc}
\hline Cause & Uncertainty & Correction \\
\hline angular dependence & $\pm 3.2 \%$ & $0.0 \%$ \\
uncertainty in $S$ & $\pm 1.2 \%$ & $0.0 \%$ \\
beam spot position & $\pm 0.5 \%$ & $+0.5 \%$ \\
remaining background & $\pm 0.25 \%$ & $-0.25 \%$ \\
total & $\pm 3.5 \%$ & $0.25 \%$ \\
\hline
\end{tabular}

the final result.

- Backgrounds: Residual background from multihadronic events in the three-prong sample is estimated from Monte Carlo studies to be $0.25 \pm 0.25 \%$. This is a correction to the final result and a contribution to its systematic uncertainty.

- Effect of cuts: Tightening the vertex $\chi^{2}$ probability cut and the decay length error cut had a negligible effect on the result, as did widening or shortening the decay length window.

The various sources of systematic uncertainties are summarized in Table 3. It is possible for the result to be biased by the selection cuts. For example, were hidden variables to favour specific regions of phase space, they could also favour specific three-prong opening angles. Since decay length errors are correlated with decay opening angles, any deviation in the zero decay length region could be removed by a cut on this error. We conclude that the formation of bias through the introduction of cuts is possible, but since their evaluation would be uncertain and model dependent, we have not considered such biases when estimating the errors. Another possibility for the introduction of a bias is that a deviation formed by hidden variables favouring small decay opening angles (corresponding to large errors on the decay length) would not be detected by the fit since such events are given a smaller weight. So, the limit resulting from this work is only valid if the error distribution of events due to unrelaxed hidden variables is the same as the normal error
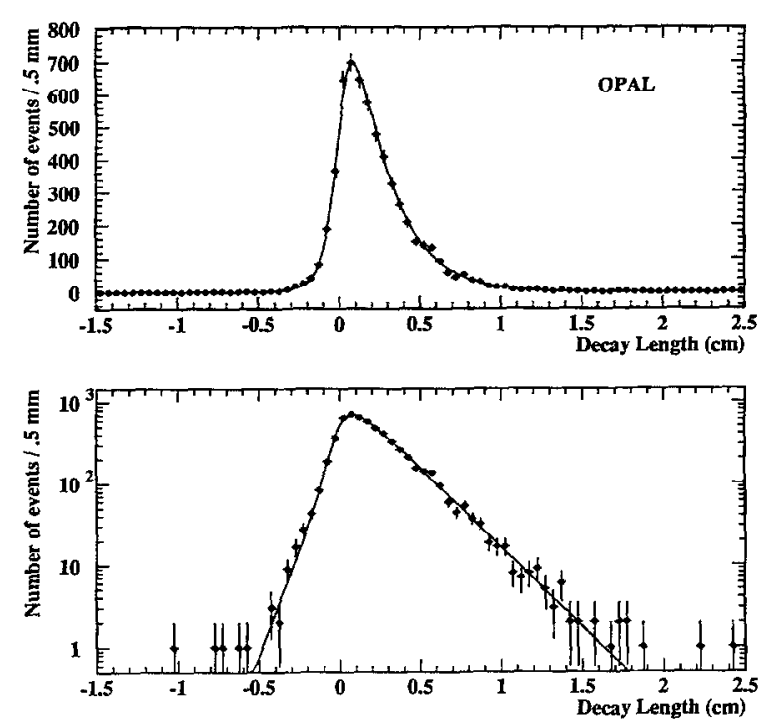

Fig. 1. Decay length distributions for the data collected in 1992 and 1993. The solid line represents the maximum likelihood three-parameter fit.

\section{distribution.}

The fit to real data, after applying the above correction, is presented in Table 4 . For completeness, although the fit procedure is a maximum likelihood one using the full window $[-15,25 \mathrm{~mm}]$, we quote the $\chi^{2}$ as calculated for the 11 bins in the $[-2.5,3.0 \mathrm{~mm}]$ decay length window, which is the most sensitive to the signal. The two-parameter fit yields a lifetime of $290.7 \pm 3.9 \mathrm{fs}$, in agreement with that presented in [7]. The three-parameter fit is presented in Fig. 1. We show in Fig. 2 the data compared with the predictions of the two-parameter likelihood fit of the lifetime and scale factor, setting the deviation $D$ to $0 \%$ (represented by the zero line of the plot) $,-3 \%,+1.1 \%$ (result of the three-parameter fit), and $+5 \%$.

In conclusion, we have searched for a deviation from exponential behaviour in the zero decay length region of the three-prong decay length distribution of the $\tau$ lepton. The analysis is based on a three-parameter

Table 4

Results of the 2 and 3-parameter fits to the data

\begin{tabular}{lllll}
\hline & $L(\mathrm{~mm})$ & $S$ & $D(\%)$ & $\chi^{2}$ per degree of freedorn \\
\hline 2-parameter fit & $2.219 \pm 0.030$ & $0.952 \pm 0.020$ & & $6.9 / 9$ \\
3-parameter fit & $2.236 \pm 0.039$ & $0.944 \pm 0.022$ & $1.1 \pm 1.4$ & $5.7 / 8$
\end{tabular}




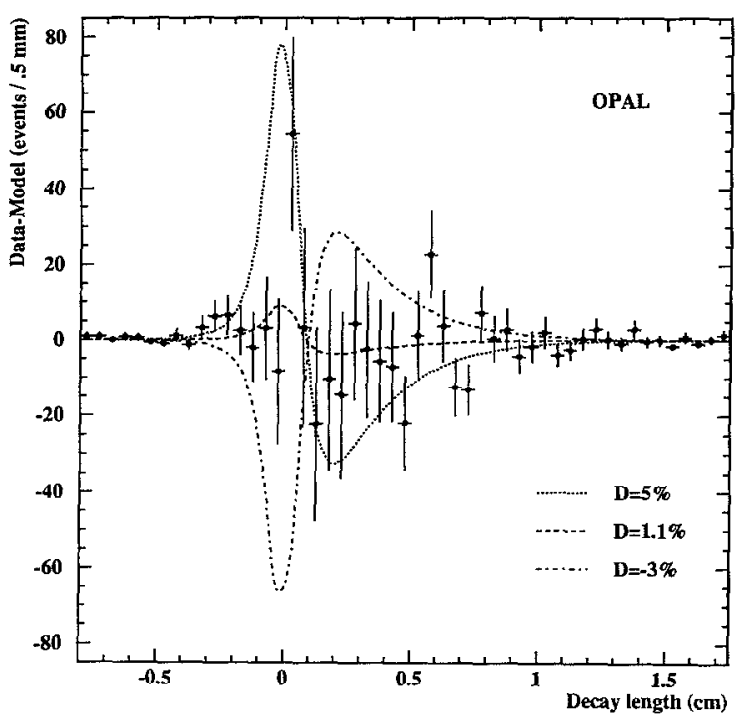

Fig. 2. Residual distributions. The points with error bars represent the residual difference (data - model) between the data and the two-parameter curve of the fitted value of $L$ and $S$ with the deviation parameter $D$ set to 0 . The lines represent the residual differences with respect to zero deviation expected for various values of the deviation parameter $D:-3 \%$ (dot-dashed line), $+1.1 \%$ (result of the fit, dashed line), and $+5 \%$ (dotted line).

fit, where the third free parameter is a measure of the excess or depletion of events in the zero decay length region. An analysis of $5843 \tau \rightarrow$ three-prong decays results in a deviation from an exponential distribution which is consistent with zero. It is expressed as the relative weight of the excess at zero decay length with respect to the number of decays present within the exponential form, and is measured to be $1.1 \% \pm 1.4 \% \pm$ $3.5 \%$. This leads to an upper limit of $8.5 \%$ and a lower limit of $-6.3 \%$ at the $95 \%$ confidence level.

It is a pleasure to thank the SL Division for the efficient operation of the LEP accelerator, the precise information on the absolute energy, and their continuing close cooperation with our experimental group. In addition to the support staff at our own institutions we are pleased to acknowledge the Department of Energy, USA,
National Science Foundation, USA,

Particle Physics and Astronomy Research Council, UK,

Natural Sciences and Engineering Research Council, Canada,

Fussefeld Foundation,

Israel Ministry of Science,

Israel Science Foundation, administered by the Israel Academy of Science and Humanities,

Minerva Gesellschaft,

Japanese Ministry of Education, Science and Culture (the Monbusho) and a grant under the Monbusho International Science Research Program,

German Israeli Bi-national Science Foundation (GIF),

Direction des Sciences de la Matière du Commissariat à l'Energie Atomique, France,

Bundesministerium für Forschung und Technologie, Germany,

National Research Council of Canada,

A.P. Sloan Foundation and Junta Nacional de Investigação Científica e Tecnológica, Portugal.

Hungarian Foundation for Scientific Research, OTKA T-016660.

\section{References}

[1] C.B. Chiu, E.C.G. Sudarshan and B. Mirsa, Phys. Rev. D 162 (1977) 520; see also, Quantum Mechanics by K. Gottfried, Vol, 1, p. 143.

[2] E.B. Norman et al., Phys. Rev. Lett. 60-22 (1988) 2246, and references therein.

[3] D. Bohm and J. Bub, Kev. Mod. Phys. 38-3 (1966) 453.

[4] R. Folman, Found. Phys. Lett. 7-2 (1994) 191.

[5] OPAL Collaboration, K. Ahmet et al., Nucl. Instr. and Meth. A 305 (1991) 275.

[6] P.P. Allport et al., Nucl. Instr. and Meth. A 324 (1993) 34; A 346 (1994) 476.

[7] OPAL Collaboration, P.D. Acton et al., Z. Phys. C 59 (1993) 183;

OPAL Collaboration, R. Akers et al., Phys. Lett. B 338 ( 1994) 497. 